\title{
Drying of Foam under Microgravity Conditions
}

\author{
Nektaria Koursari ${ }^{1}$ (D) $\cdot$ Omid Arjmandi-Tash ${ }^{2} \cdot$ Anna Trybala $^{1} \cdot$ Victor M. Starov ${ }^{1}$
}

Received: 1 March 2019 / Accepted: 10 June 2019/Published online: 8 July 2019

(C) The Author(s) 2019

\begin{abstract}
Foams have recently been characterised as ideal products for pharmaceutical and topical use applications for the delivery of topical active agents. Foams are usually produced in a wet form but in a number of applications moderately dry foams are required. Drying of foam under terrestrial conditions proceeds under the action of gravity, which is impossible under microgravity condition. Below a new method of drying foams under microgravity condition is suggested. According to this method foam should be placed on a porous support, which will absorb the liquid from foam based on capillary forces only. The final liquid content inside the foam can be achieved by a proper selection of the porous support. The suggested method allows drying foams under microgravity conditions. Interaction of foams with porous support under terrestrial conditions was developed only recently and theoretically investigated (Arjmandi-Tash, O.; Kovalchuk, N.; Trybala, A.; Starov, V. Foam Drainage Placed on a Porous Substrate. Soft Matter 2015, 11 (18), 3643-3652) followed by a theory of foam drainage on thin porous substrates (Koursari, N.; Arjmandi-Tash, O.; Johnson, P.; Trybala, A.; Starov, M. V. Foam Drainage Placed on Thin Porous Substrate. Soft Matter, 2019, (submitted)), where rate of drainage, radius of the wetted area inside the porous layer and other characteristics of the process were predicted. The latter model is modified below to investigate foam drying under microgravity conditions. Model predictions are compared with experimental observations for foam created using Triton X-100 at concentrations above CMC. Wetted radius inside the porous substrate was measured and results were compered to model predictions. Experimental observations for spreading area versus time show reasonable agreement with theoretical predictions for all investigated systems.
\end{abstract}

Keywords Microgravity $\cdot$ Foam drying $\cdot$ Porous substrate $\cdot$ Capillarity

\section{Nomenclature}

$\Delta \quad$ Thickness of porous layer

$R(t) \quad$ Radius of the wetted area inside the porous layer

$R_{c} \quad$ Radius of column

$R_{b} \quad$ Bubble radius

$R_{p b} \quad$ Plateau border radius

$R_{p m} \quad$ Average pore radius of porous media

$\varphi \quad$ Liquid volume fraction

This article belongs to the Topical Collection: Thirty Years of Microgravity Research - A Topical Collection Dedicated to J. C. Legros Guest Editor: Valentina Shevtsova

Nektaria Koursari

N.Koursari@lboro.ac.uk

1 Department of Chemical Engineering, Loughborough University, Loughborough LE11 3TU, UK

2 Department of Chemical and Biological Engineering, University of Sheffield, Sheffield S10 2TN, UK $\varphi_{i} \quad$ Initial liquid volume fraction

$\varphi_{f} \quad$ Final Liquid volume fraction at foam/ porous layer interface

$\varphi_{c r} \quad$ Critical liquid volume fraction

$\varphi_{\max }$ Maximum liquid volume fraction

$\mu \quad$ Dynamic viscosity of liquid

$\mu_{s} \quad$ Surface viscosity

$\gamma \quad$ Liquid-air interfacial tension

$\rho \quad$ Liquid density

g Gravity acceleration

$n_{p} \quad$ Number of Plateau borders per bubble

$C^{2} \quad 0.161$ (constant)

c Velocity coefficient, $c=0.5169$ for foams produced by Newtonian solutions

$C_{1} \quad$ Geometrical coefficient, $\sim 0.378-0.972$ for foam with structures between bcc and fcc and bubbles of same size.

$\zeta \quad$ The dimensionless horizontal scale

$\zeta_{H} \quad$ The dimensionless length of the column

$\zeta_{1} \quad$ The dimensionless position of the left-hand side boundary of the foam 
$\zeta_{2} \quad$ The dimensionless position of the foam/porous layer boundary

$\zeta_{\Delta} \quad \Delta / z_{0}$

$\tau \quad$ The dimensionless time

$l$ The dimensionless radius of the absorbed area into the porous area

$l_{c} \quad$ The dimensionless radius of the column

Bo Bond number

$P_{b} \quad$ Pressure inside bubbles

$P_{c} \quad$ Capillary pressure

$P_{p m} \quad$ Mean capillary pressure inside capillaries in the porous layer.

$\alpha \quad$ Ratio of capillary pressure in porous layer to capillary pressure in the bubbles

$v \quad$ Velocity at circular edge of the wetted region inside the porous layer

$\varepsilon \quad$ Porosity of porous layer

$Q \quad$ Total volumetric flux through the Plateau borders

$Q_{f} \quad$ Flux of the liquid at foam/porous layer interface

$Q_{p m} \quad$ Flux of the liquid inside the porous layer

$V_{f} \quad$ Volume of liquid inside the foam

\section{Introduction}

Foam is a colloidal dispersion where gas bubbles are dispersed in a continuous liquid phase. The bubbles, referred to as the dispersed phase, are polyhedral and are separated by thin films called lamella. The border where lamella (soap films) meet between three gas bubbles is referred to as Plateau border (channel), which join by a factor of four in regions called nodes (vertices) (Carrier et al. 2002; Schramm 1994). Foam bubbles are characterised by their bubble radius. Bubble radius and other characteristic parameters, i.e. interface elasticity, liquid density and viscosity, liquid volume fraction, gravity and capillarity determine the foam stability and the properties of the foam (Schramm 1994). The liquid volume fraction, $\varphi$, varies between $\sim 1 \%$ and $\sim 35 \%$, while most of the liquid is concentrated in the Plateau borders at reasonably low liquid volume fraction below $\sim 12 \%$, which is considered below. In this case most of the liquid in foams is accumulated inside the Plateau borders. However, the suggested method of drying foam at microgravity conditions is applicable at any liquid volume fraction below $\sim 35 \%$.

The term 'foam stability' refers to the stability of the foam films between gas bubbles which can be controlled by the addition of foaming agents, i.e. surfactants, polymers or proteins that form mono- or multi-layers at the lamella/air and bubble/air interfaces, reducing the surface tension of the multiphase system (Koehler et al. 2000; Schramm 2005).

Foam have a variety of industrial and domestic applications and are closely related to the production of a number of wellknown products, i.e. shaving foams, firefighting foams, personal care products, medical products, structural material and processes such as floatation and mineral separation (Schramm 2005). In hydrocarbon industry foams are also utilised at all stages in the oil recovery process including well drilling and oil well production (Hart et al. 1997).

For liquid foams under terrestrial conditions the liquid flows through Plateau regions due to effect of gravity and capillarity forces. The action of gravity and capillarity result in the alteration of the local liquid volume fraction inside the foam body. When gravity and capillarity equilibrate, then steady state is reached (Koursari, N.; Arjmandi-Tash, O.; Johnson, P.; Trybala, A.; Starov, M. V. Foam Drainage Placed on Thin Porous Substrate. Soft Matter, 2019, (submitted)). The liquid flow though Plateau borders, films and nodes is referred to as foam drainage. Gravitational drainage causes the liquid to drain out of the foam body to the lower layers following the gravity direction (Exerowa and Kruglyakov 1998). The drainage equations were introduced in (Goldfarb et al. 1987) and (Goldshtein et al. 1996; Verbist et al. 1996) based on continuity and pressure balance considerations taking into account flow inside Plateau borders only. Below we consider moderately dry foams with initial liquid volume fraction below $12 \%$ and include all liquid into Plateau borders (PB), that is the whole liquid is concentrated inside the PBs. Estimations below (see Eq. (4)) based on calculations by (Wang Narsimhan 2006) show that the influence of surface viscosity can be neglected and no-slip boundary conditions can be adopted on the PB surfaces.

The drainage equation has been solved analytically for a multiplicity of cases including forced drainage (Verbist et al. 1996), gravity free drainage (Sun et al. 2008) and free drainage (Cox et al. 2000).

However, gravitational drainage is not always desirable for a number of applications such as the fabrication of metallic foams (Banhart 1999; Banhart and Weaire 2002), oil recovery techniques (Hart et al. 1997), floatation and pumping (Vandewalle et al. 2011). Gravity affects the foam formation, its evolution and stability by causing flow of liquid from higher to lower layers of the foam, while microgravity conditions allow the exclusive investigation of capillary flows only. Therefore, investigating the behaviour of foams under microgravity conditions allows overcoming the instability barriers imposed when gravity forces are present (Caps et al. 2003).

Microgravity is not only desirable for the investigation of capillary drainage but also for several research studies leading to optimisation of terrestrial processes and product quality improvement. In fluid-science experiments effects such as convection, sedimentation, stratification and fluid static pressure can be eliminated under microgravity conditions and therefore allows the investigation of fluid-dynamic effects normally affected by gravitation (Dubois et al. 1999). Other applications of microgravity investigations include frontal displacement of fluids (Smirnov et al. 2005) where instability of 
the interface could lead to the creation of gas 'fingers' penetrating the bulk fluid, capillary driven filtration in porous media (Smirnov et al. 2003), Soret Coefficients of Organic Solutions measured in the Microgravity (Van Vaerenbergh and Legros 1998), thermodiffusion microgravity measurements of ternary mixtures (Touzet et al. 2011) measurements of thermo- and diffusio-phoretic velocities of aerosol particles (particle motion), which play a significant role in the scavenging of aerosols due to crystal growth or evaporation (Prodi et al. 2006). Microgravity conditions also benefit the investigation of bubble dynamics during degassing of liquids for applications heat pumps, heat exchangers, cooling systems, food technology and human physiology (Divinis et al. 2006), and microgravity investigations of drainage in porous media (Smirnov et al. 2004).

Recently, microgravity experiments have been performed (Caps et al. 2003), in parabolic flights which allow $20 \mathrm{~s}$ in microgravity established at the top of the parabolic trajectory and the so-called "capillary imbibition or capillary wetting" was investigated. Cox et al. 2004 complemented on the latter experimental study by modifying the standard drainage models for the microgravity case (Cox et al. 2004). The authors considered two equations based upon i) Poiseuille flow through Plateau borders and ii) assuming plug flow in the Plateau borders and proved that the liquid move upwards into the foam body (Cox et al. 2004). Experiments and simulations studying liquid flow into the foam body were also performed by SaintJalmes et al. (2006), where three parabolic flight campaigns were performed under the supervision of European Space Agency (ESA). The purpose of the campaigns was to gain information and understanding of the foam structure, stability, rheology, the liquid distribution and the way the liquid propagates between the different structure layers in microgravity conditions (Saint-Jalmes et al. 2006).

Among the most well-known microgravity related research tasks are RADIOUS (research associations for the development of industrial use of space), dedicated to the environment and energy, C-CORE (Consortium of Industrial Research in the use of space) focusing in fluid physics experiments and MIRROR (Microgravity, Industry Related research for oil recovery) focusing on the study of diffusion coefficient of crude oil, foam stability in microgravity and capillary flow in porous media. In the case of oil industry related subjects, foams could be desirable or undesirable depending upon their intensive use (Hart et al. 1997). As part of the latter programme, foam stability experiments were performed aiming to provide information for the development of the oil recovery techniques often challenged by the effect of gravity (Hart et al. 1997).

Recently, foams have been characterised as ideal products for the delivery of topical active agents such as skin protectants, corticosteroids and local anaesthetics ideal for dermatological applications (Zhao et al. 2010). The kinetics of topical drug delivery depend upon the foam properties, the porous material and the interaction of the foam/thin porous layer system. Due to the increasing interest in liquid drainage of foams on porous material (Arjmandi-Tash et al. 2015; Bureiko et al. 2015; Farajzadeh et al. 2012) a theory of foam drainage placed on thin porous substrate was recently developed under terrestrial gravity conditions (Koursari, N.; Arjmandi-Tash, O.; Johnson, P.; Trybala, A.; Starov, M. V. Foam Drainage Placed on Thin Porous Substrate. Soft Matter, 2019, (submitted)). In this study drainage process was considered as a combination of drainage caused by gravity/ capillary action and a capillary suction applied by the pores from the porous substrate. The rate of drainage, radius of the wetted area inside the porous material and other characteristics of the process were theoretically predicted and compared with experimental observations on various foam-thin substrates systems.

Below the previously developed theory of foam drainage on thin porous material (Koursari, N.; Arjmandi-Tash, O.; Johnson, P.; Trybala, A.; Starov, M. V. Foam Drainage Placed on Thin Porous Substrate. Soft Matter, 2019, (submitted)), has been adapted and modified to be applied to foams drying on porous material in the absence of gravitational field. Experimental observations are compared with theoretical predictions for surfactant solutions Triton-X$10050 \mathrm{CMC}$ and $20 \mathrm{CMC}$ on four types of filter papers, i.e. grade $1,3,5$ and 601 .

The experiments were conducted in the laboratory while the effect of gravity was eliminated by using a foam column placed horizontally, attached to one end on a thin porous substrate (Fig. 1).

\section{Theory}

1. Let us consider foam produced from a Newtonian surfactant solution in a column of length $H$ attached to a thin porous layer (Fig. 1). All bubbles are supposed to be of a uniform size and do not change size over duration of the experiments. Liquid flow inside the foam, caused by a capillary suction applied by the pores of the porous layer, occurs in the horizontal direction along the co-ordinate axis $\mathrm{z}$ directed horizontally, with $z=0$ at the left-hand side of the column.

The flow inside the foam is assumed to occur inside the Plateau regions only and the contribution of the liquid flow in the films and nodes is neglected. In this case the drainage kinetics can be described by the following equation (Arjmandi-Tash et al. 2017; Arjmandi-Tash et al. 2015; 
Fig. 1 Schematic presentation of the foam in horizontal position attached on one-side to a thin porous substrate. 1 - foam; 2 porous layer of thickness $\Delta ; \mathrm{R}(\mathrm{t})$ is the radius of the wetted area inside the porous layer 3- digital camera $1 ; 4$ - digital camera $2 ; 5$ interface to PC from camera $1 ; 6$ interface to PC from camera $2 ; 7$ clamp

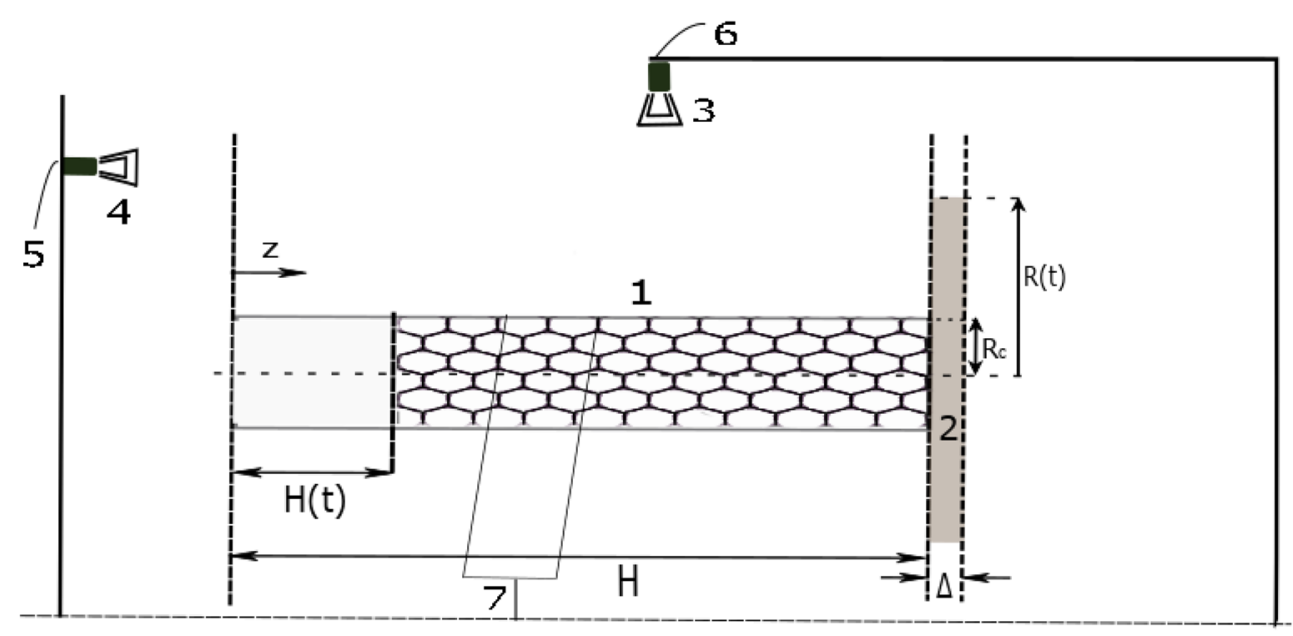

Bureiko et al. 2015; Kruglyakov et al. 2008; Wang and Narsimhan 2006; Wierenga and Gruppen 2010).

$\frac{d \varphi}{d t}+\frac{d Q}{d z}=0$,

where $Q$ is the flow rate expressed as follows:

$Q=\left(\frac{C_{1}^{\frac{1}{2}} \rho g}{\mu \beta} R_{b}^{2} \frac{\varphi^{2}}{(1-\varphi)^{\frac{1}{3}}}-\frac{C \gamma}{2 \mu \beta} R_{b} \frac{\varphi^{\frac{1}{2}}\left(1-\frac{\varphi}{3}\right)}{(1-\varphi)} \frac{d \varphi}{d z}\right)$

Under microgravity conditions, $g=0$, and Eq. (2) can be rewritten as:

$Q=\left(-\frac{C \gamma}{2 \mu \beta} R_{b} \frac{\varphi^{\frac{1}{2}}\left(1-\frac{\varphi}{3}\right)}{(1-\varphi)} \frac{d \varphi}{d z}\right)$

and

$\frac{1}{\beta}=\left(c \frac{1}{3^{\frac{3}{2}} 5} \frac{1}{n_{p}^{\frac{1}{2}} \delta^{\frac{3}{2}} \pi^{\frac{1}{2}}}\right)$

According to (Wang and Narsimhan 2006) the influence of surface viscocity can be taken into account as follows: in Eq. (4) the coefficient $c$ should be presented as $c=a+b$ $\left(\frac{R_{b} \mu}{\mu_{s}}\right), a=0.5169 ; b=0.5442$, where $\mu$ is liquid viscosity, $R_{b}$ is radius of bubbles, and $\mu_{s}$ is surface viscosity. $\left(\mu_{s} / R_{b} \mu\right)$ is Bousinesq number. In the case under consideration $\frac{\mu \cdot R_{b}}{\mu_{s}} \sim 2.2 x$ $10^{-2} \ll 1$. The latter means that the influence of surface viscosity can be neglected in Eq. (4) and no-slip boundary condition are adopted on the surface of PB. Substituting (3) into (1) results in:

$$
\frac{\partial \varphi}{\partial \mathrm{t}}+\frac{\partial\left(-\frac{C \gamma}{2 \mu \beta} R_{b} \frac{\varphi^{\frac{1}{2}}\left(1-\frac{\varphi}{3}\right)}{(1-\varphi)} \frac{d \varphi}{d z}\right)}{\partial z}=0
$$

In the above equations, $\varphi$ is the liquid volume fraction; $\mu$ is the dynamic viscosity of the liquid; $\gamma$ is the liquid-air interfacial tension; $R_{b}$ is the radius of bubbles; $\rho$ and $g$ are the liquid density and the gravity acceleration, respectively; $n_{p}$ is the number of Plateau borders per bubble; $\beta$ is a coefficient (Arjmandi-Tash et al. 2017; Wang and Narsimhan 2006); $C^{2}=\sqrt{3}-\pi / 2 \sim 0.161 ; c=0.5169$ is the velocity coefficient (Wang and Narsimhan 2006) foams produced from Newtonian solutions; $C_{1}$ is a geometrical coefficient expressed as $C_{1}=\frac{4 \pi}{\left(3 n_{p} \delta\right)} \sim 0.378-0.972$ for a foam with structures between bcc (body-centred cubic) and fcc (face-centred cubic) and bubbles of the same size $\left(\delta=0.718-1.108, \mathrm{n}_{\mathrm{p}}=6\right.$ -10 )(Weaire and Hutzler 1999). The non-slip boundary condition on the liquid-air interface of Plateau borders are adopted below. For this purpose the Triton $-\mathrm{X} 100$ surfactant was selected, which has a relatively high surface viscosity.

Let us introduce the following dimensionless variable and co-ordinate: $\zeta \rightarrow \frac{z}{z_{0}}, \tau \rightarrow \frac{t}{t_{0}}$, where $z_{0}$ and $t_{0}$ the characteristic scale and time respectively.

Non-dimensonalisation of Eq. (5) results in:

$\frac{\partial \varphi}{\partial \tau}+\frac{\partial\left(-\frac{t_{0}}{z_{0}^{2}} \cdot \frac{C \cdot \gamma}{2 \cdot \mu \cdot \beta} \cdot R_{b} \frac{\varphi^{\frac{1}{2}}\left(1-\frac{\varphi}{3}\right)}{(1-\varphi)} \cdot \frac{d \varphi}{d \zeta}\right)}{\partial \zeta}=0$

If $z_{0}=H[\mathrm{~m}]$ is the characteristic scale, then from Eq. (6) considering Eq. (7): 
$\frac{t_{0}}{z_{0}^{2}} \cdot \frac{C \cdot \gamma}{2 \cdot \mu \cdot \beta} \cdot R_{b}=1$

The expression of the characteristic time is as follows:

$t_{0}=\frac{z_{0}^{2} \cdot 2 \cdot \mu \cdot \beta}{C \cdot \gamma \cdot R_{b}}=\frac{H^{2} \cdot 2 \cdot \mu \cdot \beta}{C \cdot \gamma \cdot R_{b}}$

Substitution of $t_{0}$ into Eq. (6) results in

$\frac{d \varphi}{d \tau}+\frac{d}{d \zeta}\left(\frac{-\varphi^{\frac{1}{2}}\left(1-\frac{\varphi}{3}\right)}{(1-\varphi)} \frac{d \varphi}{d \zeta}\right)=0$

which is a non-linear diffusion equation. Assuming that the liquid films between bubbles in foam are flat, i.e. $R_{b} \gg R_{p b}$, (see Fig. 2) the pressure in all bubbles is identical (ArjmandiTash et al. 2015; Bureiko et al. 2015; Grassia et al. 2001) and equals to:

$P_{b}=\frac{4 \gamma}{R_{b}}$

$P_{c}=\frac{\gamma}{R_{p b}}$

where $P_{c}$ is the capillary pressure and $\gamma$ the liquid-air interfacial tension.
According to (Weaire and Hutzler 1999)

$C^{2} R_{p b}^{2}=C_{1} R_{b}^{2} \frac{\varphi}{(1-\varphi)^{\frac{2}{3}}}$

From Eqs. (10-12) the pressure in the Plateau border is:

$P_{p b}(z, t)=P_{b}-P_{c}=\frac{\gamma}{R_{b}}\left(4-\frac{C}{C_{1}^{\frac{1}{2}}} \frac{(1-\varphi(z, t))^{\frac{1}{3}}}{\varphi(z, t)^{\frac{1}{2}}}\right)$.

Let us introduce $P_{p m}$ as the mean capillary pressure inside capillaries inside the porous layer, which can be estimated by $P_{p m} \sim \frac{n \gamma}{R_{p m}}$, where $R_{p m}$ is radius of pores, $n=1$ in the case of porous media built by cylinders (for example hair) and $n=2$ in the case of two-dimensional geometry. Accordingly, the pressure difference, which results in the liquid flow from the Plateau channel into the porous layer at the foam/porous layer interface, is:

$$
\begin{aligned}
\Delta P=P_{p b}-P_{p m}= & \frac{\gamma}{R_{b}}\left(4-\frac{C}{C_{1}^{\frac{1}{2}}} \frac{(1-\varphi(z=H, t))^{\frac{1}{3}}}{\varphi(z=H, t)^{\frac{1}{2}}}\right) \\
& +\frac{n \gamma}{R_{p m}} .
\end{aligned}
$$

Fig. 2 Schematic presentation of a generalised, homogenous foam system formed of identical gas bubbles of radius $R_{b}$ in a continuous liquid phase and liquid filled channels and nodes. The connection of three lamellae is referred to as Plateau border (concave triangles) of radius $\mathrm{R}_{\mathrm{pb}}$, being the radius of curvature of the sides. (Koursari, N.;

Arjmandi-Tash, O.; Johnson, P.; Trybala, A.; Starov, M. V. Foam Drainage Placed on Thin Porous Substrate. Soft Matter, 2019, (submitted))

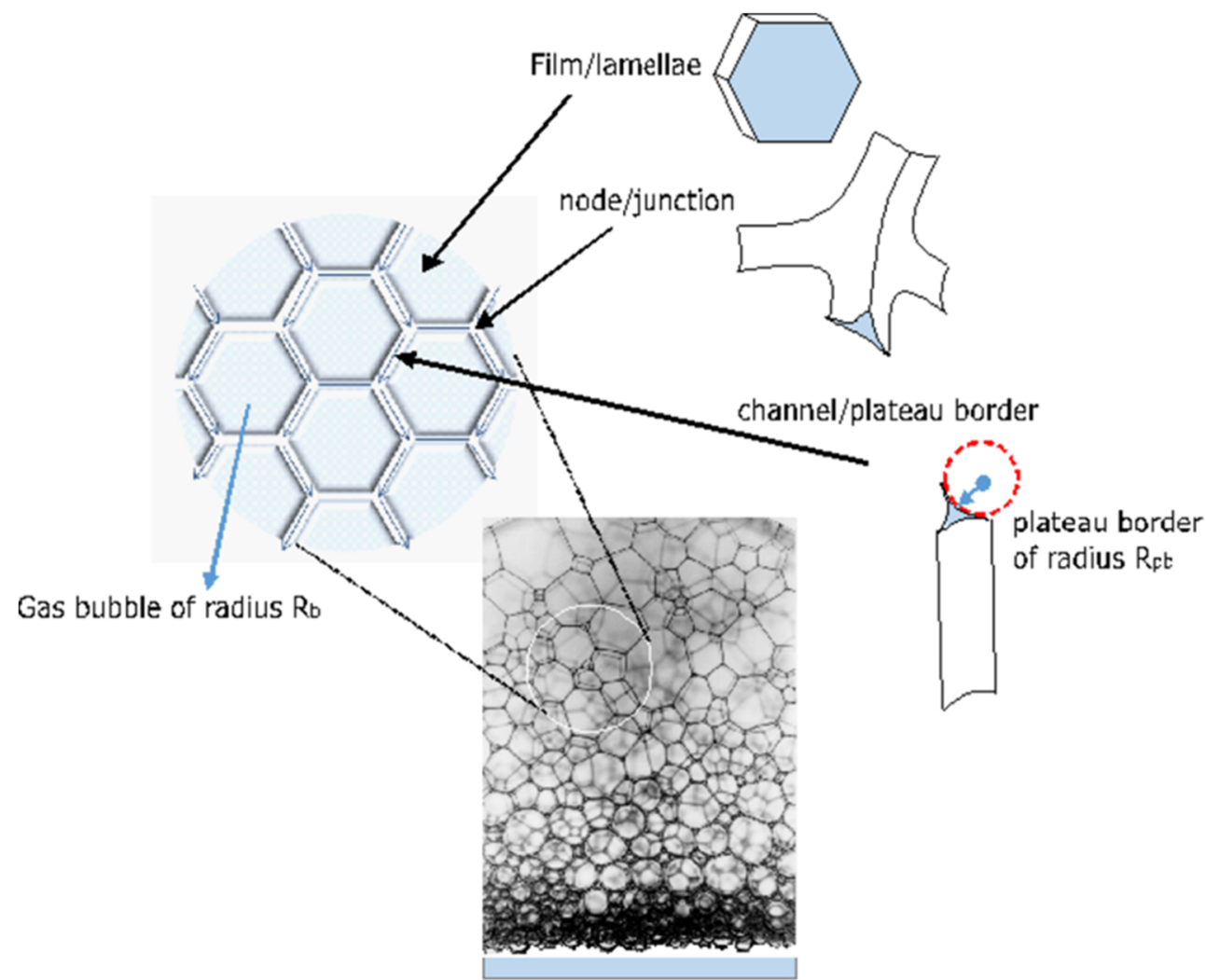


If $\Delta P>0$, then liquid from the Plateau border will penetrate the porous layer. However, if $\Delta P<0$, then penetration will stop. Let us introduce the final liquid content, $\varphi_{f}$, as the liquid volume fraction at the foam/porous layer interface at which the pressure inside the Plateau channels and the pressure inside the porous layer equilibrate. The consideration above and Eq. (11) determine the end of the drainage process and the final liquid content at the foam/porous substrate interface, $\varphi_{f}$, when $\Delta P=0$ :

$\frac{\gamma}{R_{b}}\left(4-\frac{C}{C_{1}^{\frac{1}{2}}} \frac{(1-\varphi(z=H, t))^{\frac{1}{3}}}{\varphi(z=H, t)^{\frac{1}{2}}}\right)+\frac{n \gamma}{R_{p m}}=0$.

Hence,

$$
\frac{\varphi_{f}}{\left(1-\varphi_{f}\right)^{\frac{2}{3}}}=\frac{\left(\frac{C^{2}}{16 C_{1}}\right)}{\left(1+\frac{n R_{b}}{4 R_{p m}}\right)^{2}}
$$

If we introduce $\alpha$ as a ratio of capillary pressure in the porous layer to capillary pressure in the bubbles:

$$
\alpha=\frac{\Delta P_{\text {capin substrate }}}{\Delta P_{\text {capin bubbles }}}=\frac{\frac{n \gamma}{R_{p m}}}{\frac{4 \gamma}{R_{b}}}=\frac{n R_{b}}{4 R_{p m}}
$$

and taking into account that $\varphi_{f}<<1$ then, Eq. (16) can be rewritten as:

$$
\varphi_{f}=\frac{\left(\frac{C^{2}}{16 C_{1}}\right)}{(1+\alpha)^{2}}
$$

According to the definition $\varphi_{f}=\frac{\left(\frac{C^{2}}{16 C_{1}}\right)}{\left(1+\frac{2 R_{b}}{4 R_{p m}}\right)^{2}}<\varphi_{\max }$ or $\left[\frac{\left(\frac{C^{2}}{16 C_{1}}\right)}{\varphi_{\max }}\right]^{-\frac{1}{2}}<\left(1+\frac{2 R_{b}}{4 R_{p m}}\right)$. Estimations show that at $C^{2} \sim 0.161, \quad C_{1}=\frac{4 \pi}{\left(3 n_{p} \delta\right)} \sim 0.378-0.972$, and $\varphi_{\max }=$ $0.26:\left[\frac{\left(\frac{c^{2}}{16 C_{1}}\right)}{\varphi_{\max }}\right]^{-\frac{1}{2}} \sim 0.2-0.32$. The latter means that $\varphi_{f}<\varphi_{\max }$ at any size of pores and foam bubbles.

Equation (18) shows that it is possible to control the final liquid contents inside the foam simply by varying the parameter $\alpha$, which is determined by the average pore radius inside the porous layer.

Equation (16) allows calculating the final radius, of the wetted area inside the porous layer, $R_{f}$. The final volume fraction is equal to $\varphi_{f}$ and the volume of liquid inside the foam $V_{f}$ $=\pi \cdot H \cdot R_{c}^{2} \cdot \varphi_{f}$ according to the mass conservation law: $V_{f}+\pi \cdot \varepsilon \cdot \Delta \cdot R_{f}^{2}=V_{i}$, where the initial liquid volume inside the foam is: $V_{i}=\pi \cdot H \cdot R_{c}^{2} \cdot \varphi_{i}$. Substitution of expressions for initial and final volume we arrive to:

$l_{f}=\sqrt{\frac{\varphi_{i}-\varphi_{f}}{\varepsilon \cdot \Delta / H}}$

The suggested method allows (i) to vary final values of the liquid contents inside the foam, $\varphi_{f}$, by varying porosity, thickness and capillary pressure inside the porous substrate; (ii) it is possible to stop imbibition into the porous layer at any moment simply by removing the porous layer. It will allow to reach any pre-scribed value of the remaining liquid inside the foam in between $\varphi_{i}$ and $\varphi_{f}$.

Equation (18) and the previous consideration shows that the drainage of foam placed on the porous substrates under microgravity conditions is substantially different from that in the case of free drainage or drainage of foam placed on a nonporous substrate: in the case of drainage of foam placed on porous substrate the final liquid volume fraction at the foam/porous substrate interface is much lower as compared with the case of free drainage, when this volume fraction reaches the maximum possible value.

\section{Liquid imbibition into porous layer}

It is assumed below, according to (Starov et al. 2002), that the porous layer is thin enough and the time for its saturation in the vertical direction can be neglected relative to other time scales of the process.

The liquid flow inside the porous layer in the radial direction obeys the Darcy's law:

$\frac{1}{r} \frac{d}{d r}(r v)=0, v=\frac{K_{p}}{\mu}\left|\frac{d p}{d r}\right|$

Solution of the latter equations is:

$p=-\left(\frac{\mu}{K_{p}}\right) \ln (r)+B, v=\frac{C}{r}$

where $B$ and $C$ are integration constants, which should be determined using the boundary conditions for the pressure at the column edge, $r=R_{c}$, and at the circular edge of the wetted region inside the porous layer, $r=R(t)$ (Starov et al. 2002). 
The boundary condition at the circular edge of the wetted region inside the porous layer is expressed as:

$P=-P_{p m}, r=R(t)$,

where $P_{p m} \approx \frac{n \gamma}{R_{p m}}$ is the capillary pressure inside the pores of the porous layer, and $R_{p m}$ is a characteristic scale of the pore radii inside the porous layer.

The other boundary condition is written as:

$P=\frac{\gamma}{R_{b}}\left(4-\frac{C}{C_{1}^{\frac{1}{2}}} \frac{(1-\varphi(H, t))^{\frac{1}{3}}}{\varphi(H, t)^{\frac{1}{2}}}\right), r=R_{c}$.

Using the latter two boundary conditions, both integration constants, $b$ and $c$, can be determined, which gives the following expression for the radial velocity according to Eq. (21):

$v=\frac{K_{p}}{\mu} \frac{\Delta P}{r \ln \left(\frac{R}{R_{c}}\right)}$,

where $\Delta P$ is given by Eq. (14).

The velocity at the circular edge of the wetted region inside the porous layer is:

$\varepsilon \frac{d R}{d t}=\left.v\right|_{r=R}$

where $\varepsilon$ is the porosity of the porous layer. Combination of the latter two equations gives the evolution equation for $R(t)$ :

$\frac{d R}{d t}=\frac{K_{p}}{\varepsilon \mu} \frac{\Delta P}{R \ln \left(\frac{R}{R_{c}}\right)}$

Let us introduce the following dimensionless variables: $l \rightarrow$ $\frac{R}{R_{c}}, \tau_{1} \rightarrow \frac{t}{t_{00}}$ where $t_{00}$ is a characteristic time scale of the imbibition into the porous substrate and $R_{c}$ is the radius of the tube. Let us estimate $\Delta P$ according to Eqs. (14) and (18) as:

$$
\begin{aligned}
\Delta P=P_{p b}-P_{p m}= & \frac{4 \gamma}{R_{b}}\left(1-\frac{C}{4 \cdot C_{1}^{\frac{1}{2}}} \frac{(1-\varphi(z=H, t))^{\frac{1}{3}}}{\varphi(z=H, t)^{\frac{1}{2}}}\right) \\
& +\frac{n \cdot \gamma}{R_{p m}} \\
= & \frac{4 \gamma(1+\alpha)}{R_{b}}\left(1-\frac{C}{4 \cdot C_{1}^{\frac{1}{2}}(1+\alpha)} \frac{(1-\varphi(z=H, t))^{\frac{1}{3}}}{\varphi(z=H, t)^{\frac{1}{2}}}\right) \\
= & \frac{4 \gamma(1+\alpha)}{R_{b}}\left(1-\frac{\varphi_{f}^{1 / 2}}{\left(1-\varphi_{f}\right)^{1 / 3}} \frac{(1-\varphi(z=H, t))^{\frac{1}{3}}}{\varphi(z=H, t)^{\frac{1}{2}}}\right)
\end{aligned}
$$

Hence, according to Eq. (26) $t_{00}$ can be estimated as:

$t_{00}=\frac{R_{b} \varepsilon \mu R_{c}^{2}}{4 \gamma K_{p}(1+\alpha)}$

Using new variables, we can rewrite Eq. (26) as:

$\frac{d l}{d \tau_{1}}=\frac{1}{l \cdot \ln l}\left(1-\frac{\sqrt[3]{\frac{(1-\varphi(\zeta=1, \tau))}{\left(1-\varphi_{f}\right)}}}{\sqrt{\frac{\varphi(\zeta=1, \tau)}{\varphi_{f}}}}\right)$

In the latter Eq. (29) the left-hand side depends on a new time $\tau_{1}$ while the right-hand site depends on $\tau$. Relation between these two dimensionless time scales is: $\tau=\frac{t_{00}}{t_{0}} \cdot \tau_{1}$.

If we switch to the dimensionless time $\tau$ in Eq. (29), it results in:

$\frac{t_{00} \cdot d l}{t_{0} \cdot d \tau}=\frac{1}{l \cdot \ln l}\left(1-\frac{\sqrt[3]{\frac{(1-\varphi(\zeta=1, \tau))}{\left(1-\varphi_{f}\right)}}}{\sqrt{\frac{\varphi(\zeta=1, \tau)}{\varphi_{f}}}}\right)$

Let us define $\delta=\frac{t_{00}}{t_{0}}$, that is the ratio of characteristic time scale $t_{00}$, which is the time scale of the liquid imbibition into the porous layer and $t_{0}$, which is the time scale of the equilibration of liquid contents inside the foam:

$\delta \cdot \frac{d l}{d \tau}=\frac{1}{l \ln l}\left(1-\frac{\sqrt[3]{\frac{(1-\varphi(\zeta=1, \tau))}{\left(1-\varphi_{f}\right)}}}{\sqrt{\frac{\varphi(\zeta=1, \tau)}{\varphi_{f}}}}\right)$

3. Boundary conditions at the foam/porous layer interface

In our previous publication (Arjmandi-Tash et al. 2015), it was assumed that the height of the foam remains constant during the contact with the vertical porous layer and it was possible to neglect the small changes in the foam height which occurs due to the drainage of the foam. It was possible to assume because penetration of the liquid into the vertical thick porous medium was small enough. Situation is different in the case under consideration. That is, below the changes of the foam height due to the liquid drainage from the foam into the porous layer is taken into account. 
Let us consider a mass conservation law of liquid within the column and porous layer, in the case when the free liquid layer does not form at the foam/porous layer interface:

$$
\begin{aligned}
& \pi R_{c}^{2}\left(\int_{H_{1}(t)}^{H} \varphi(z, t) d z\right)+\varepsilon \Delta \pi R^{2}(t)=V_{0} \\
& =\pi R_{c}^{2} \varphi_{i}\left(H-H_{1}(0)\right)
\end{aligned}
$$

Differentiating Eq. (32) with time results in:

$$
\begin{aligned}
& \pi R_{c}^{2}\left(\int_{H_{1}(t)}^{H} \frac{d}{d t} \varphi(z, t) d z-\varphi\left(H_{1}(t), t\right) \frac{d H_{1}(t)}{d t}\right) \\
& \quad+2 \varepsilon \Delta \pi R(t) \frac{d R(t)}{d t} \\
& \quad=0
\end{aligned}
$$

Using Eqs. (1) and (2) the latter equation can be rewritten as:

$$
\begin{aligned}
& \pi R_{c}^{2}\left(Q\left(H_{1}(t)\right)-Q\left(H_{2}\right)-\varphi\left(H_{1}(t), t\right) \frac{d H_{1}(t)}{d t}\right) \\
& \quad+2 \varepsilon \Delta \pi R(t) \frac{d R(t)}{d t} \\
& \quad=0
\end{aligned}
$$

In the case under consideration formation of a free liquid layer at the foam/porous layer interface is impossible, hence, the right-hand side of the foam of the foam remains directly in contact with the porous layer and its position does not move during the drainage. Therefore, only the air/foam interface can move because of the drainage. At the left-hand side of the column there is no accumulation/source of liquid and, hence, $Q_{f}\left(H_{1}, t\right)=0$. Let us introduce the flux of the liquid at the foam/porous layer interface as:

$$
\begin{aligned}
Q_{f}(H, t) & \left.=\pi R_{c}^{2} Q(H, t)\right) \\
& =\pi R_{c}^{2}\left(-\frac{C \gamma}{2 \mu \beta} R_{b} \frac{\frac{\varphi^{\frac{1}{2}}(H, t)(1-\varphi(H, t)}{3}}{(1-\varphi(H, t))} \frac{d \varphi(H, t)}{d z}\right)
\end{aligned}
$$

and the flux of the liquid inside the porous layer as:

$Q_{p m}(t)=\frac{2 \pi \Delta K_{p}}{\mu} \frac{\left(\frac{\gamma}{R_{b}}\left(4-\frac{C}{C_{1}^{\frac{1}{2}}} \frac{(1-\varphi(H, t))^{\frac{1}{3}}}{\varphi(H, t)^{\frac{1}{2}}}\right)+\frac{n \gamma}{R_{p m}}\right)}{\ln \frac{R}{R_{c}}}$
Hence, Eq. (34) can be rewritten as:

$\frac{d H_{1}(t)}{d t}=\frac{1}{\pi R_{c}^{2} \varphi\left(H_{1}(t), t\right)}\left(Q_{p m}(t)-Q_{f}(H, t)\right)$

Using dimensionless variables and co-ordinate as before: $\zeta \rightarrow \frac{Z}{Z_{0}}, l \rightarrow \frac{R}{R_{0}}, \tau \rightarrow \frac{t}{t_{0}}$, Eq. (37) can be rewritten as:

$\frac{d \zeta_{1}(\tau)}{d \tau}=\frac{1}{\varphi\left(\zeta_{1}(\tau), \tau\right)}\left[\begin{array}{c}\left(\frac{2 \varepsilon \zeta_{\Delta}}{l_{c}^{2}}\left(\frac{1}{\ln \frac{l}{l_{c}}}\left(1-\frac{\sqrt[3]{\frac{\left(1-\varphi\left(\zeta_{2}, \tau\right)\right)}{\left(1-\varphi_{f}\right)}}}{\sqrt{\frac{\varphi\left(\zeta_{2}, \tau\right)}{\varphi_{f}}}}\right)\right)\right. \\ \left(-\frac{\varphi\left(\zeta_{2}, \tau\right)^{\frac{1}{2}}\left(1-\frac{\varphi\left(\zeta_{2}, \tau\right)}{3}\right)}{\left(1-\varphi\left(\zeta_{2}, \tau\right)\right)} \frac{d \varphi\left(\zeta_{2}, \tau\right)}{d \zeta}\right)\end{array}\right]$,

where $\zeta=\Delta / z_{0}$.

Let us consider a mass conservation law of the gas phase within the foam in the case, when there is no bubble rupture at the air/foam interface i.e. $\varphi\left(H_{1}, t\right)>\varphi_{c r}\left(\varphi_{c r}\right.$ is a critical liquid volume fraction below which the coalescence/bubble burst begins) and $Q\left(H_{1}, t\right)=0$ :

$\pi R_{c}^{2}\left(\int_{H_{1}(t)}^{H_{2}} 1-\varphi(z, t) d z\right)=$ cons

Differentiating Eq. (39) with time leads to the following expressions for the rate of the moving top boundary of the foam:

$\frac{d H_{1}(t)}{d t}=\frac{1}{\pi R_{c}^{2}\left(1-\varphi\left(H_{1}(t), t\right)\right)} Q_{f}(H, t)$

and in dimensionless form:

$\frac{d \zeta_{1}(\tau)}{d \tau}=\frac{-1}{1-\varphi\left(\zeta_{1}(\tau), \tau\right)} \frac{\varphi\left(\zeta_{2}, \tau\right)^{\frac{1}{2}}\left(1-\frac{\varphi\left(\zeta_{2}, \tau\right)}{3}\right)}{\left(1-\varphi\left(\zeta_{2}, \tau\right)\right)} \frac{d \varphi\left(\zeta_{2}, \tau\right)}{d \zeta}$

Comparison of Eqs. (37) and (40) results in the following expression for the boundary condition at the foam/porous layer interface in the case, when the free liquid layer does not form:

$Q_{f}(H, t)=\left(1-\varphi\left(H_{1}(t), t\right)\right) Q_{p m}(t)$

and in dimensionless form: 


$$
\begin{aligned}
& -\frac{\varphi\left(\zeta_{2}, \tau\right)^{\frac{1}{2}}\left(1-\frac{\varphi\left(\zeta_{2}, \tau\right)}{3}\right)}{\left(1-\varphi\left(\zeta_{2}, \tau\right)\right)} \frac{d \varphi\left(\zeta_{2}, \tau\right)}{d \zeta} \\
& \quad=\left(1-\varphi\left(\zeta_{1}(\tau), \tau\right)\right) \frac{2 \varepsilon \zeta_{\Delta}}{l_{c}^{2}}\left(\frac{1}{\ln \frac{l}{l_{c}}}\left(1-\frac{\sqrt[3]{\frac{\left(1-\varphi\left(\zeta_{2}, \tau\right)\right)}{\left(1-\phi_{f}\right)}}}{\sqrt{\frac{\varphi\left(\zeta_{2}, \tau\right)}{\varphi_{f}}}}\right)\right)
\end{aligned}
$$

Note, in the above equations we supposed that the liquid volume fraction at $z=H_{1}$ is higher than $\varphi_{c n}$, there is no bubble collapsing/coalescence at the air/foam interface and the boundary condition at the air/foam interface is zero liquid flux, i.e. $Q_{f}\left(H_{1}(t), t\right)=0$. The suggested system of Eqs. (5), (24) with boundary condition (36) at foam/porous layer interface is valid when no free liquid layer is formed at foam/porous layer interface, i.e. if $\varphi(H, t)<\varphi_{\max }$, which is always the case under microgravity conditions. In this case porous layer absorbs all liquid coming from the foam and the boundary condition at the air/foam interface moves horizontally due to liquid drainage inside the foam according to Eq. (41). This case was referred to a fast or intermediate imbibition of the liquid into the porous layer" (Arjmandi-Tash et al. 2015)' (Bureiko et al. 2015). This is the only case in the case under microgravity conditions.

\section{Experimental Procedure}

Triton-X-100 is a nonionic surfactant purchased by Sigma-Aldrich (Sigma-Aldrich (MERCK) 2019). This surfactant was selected because it shows a relatively high liquid-air surface viscosity, and, hence, is close to the adopted above non-slip boundary conditions on the surface of Plateau borders. Standard solutions of Triton $\mathrm{X}-100$ with concentration $50 \mathrm{CMC}$ and 20CMC were made. The experiment was carried out in a glass tube, $9 \mathrm{~cm}$ long with a $2.5 \mathrm{~cm}$ outer diameter and $2.1 \mathrm{~cm}$ inner diameter, with a porous substrate attached to one end. Four different filter paper grades were used as the porous substrate - Grades 1, 3, 5 and 601. The glass tube was positioned horizontally held by a clamp stand, ensuring its horizontal position using a spirit level, with one camera placed above the glass tube and a second camera facing the filter paper (Fig. 1). Parafilm was used to cover the open end of the tube to prevent loss of foam due to evaporation.

The foam of uniform bubble size was created using a squeeze bottle to initial length equal to $\mathrm{L}=6 \mathrm{~cm}$ measured considering the substrate as zero point. Two Pike Allied Vision Technologies Cameras: camera 1, Sony TV Lens 1:1.8 $16 \mathrm{~mm}$ (Fig. 1) were used for capturing images of the experiment at regular time intervals.

The images captured were analyzed by ImageJ software measuring spreading radius. It was proven that the liquid drainage in the vertical direction inside the tube was negligible over duration of the experiments. Permeability of filter papers was measured by placing a strip of each grade in water recording the changes in height. The dependency between height squared against time was plotted with the gradient representing permeability. To find the porosity the glass tube was placed vertically on each filter paper grade and filled with water to a specified height. The volume of water was then divided by the wetted area resulting to the value of porosity. Initial volume fractions for each foam solution were obtained by weighing $50 \mathrm{ml}$ of foam. The initial volume fractions of foams (see Table 2) were found by weighing a known volume of foam and dividing this weight by the volume of the foam and the liquid density, that is, $\varphi_{i}=\frac{M_{i}}{\rho V_{i}}$, where $\mathrm{M}_{\mathrm{i}}$ and $\mathrm{V}_{\mathrm{i}}$ are initial weight and volume of the foam respectively; $\rho$ is the liquid density.

Each experiment repeated at least 2-3 times. The variation between the experimental data values was quantified by standard deviation and presented in Figs. 4 and 5.

Initial liquid volume fraction for surfactants Triton X-100$50 \mathrm{CMC}$ and Triton X-100 - 20 CMC was $8.56 \%$ and $10 \%$ respectively, which are below a critical volume fraction of $\sim 12 \%$, when the suggested theoretical approach could become questionable.

Table 1 shows that the thickness of the filter paper was varied more than twice, the pore radius was varied more than three times, porosity varied twice and the permeability varied more than 10 time for different filter papers used.
Table 1 Filter Paper Properties, obtained from Whatman filter paper guide (Camlab 2019; Sigma-Aldrich (MERCK) n.d.)

\begin{tabular}{lllll}
\hline Filter Paper Grade (FPG) & 1 & 3 & 5 & 601 \\
\hline Thickness (m) & $1.8 \times 10^{-4}$ & $3.9 \times 10^{-4}$ & $2 \times 10^{-4}$ & $1.6 \times 10^{-4}$ \\
Average Pore Radius (m) & $5.5 \times 10^{-6}$ & $3.0 \times 10^{-6}$ & $1.25 \times 10^{-6}$ & $5.0 \times 10^{-6}$ \\
Porosity & 0.48 & 0.77 & 0.54 & 0.90 \\
Permeability $\left(\mathrm{m}^{2}\right)$ & $9.81 \times 10^{-14}$ & $1.57 \times 10^{-13}$ & $1.50 \times 10^{-14}$ & $1.67 \times 10^{-13}$ \\
\hline
\end{tabular}




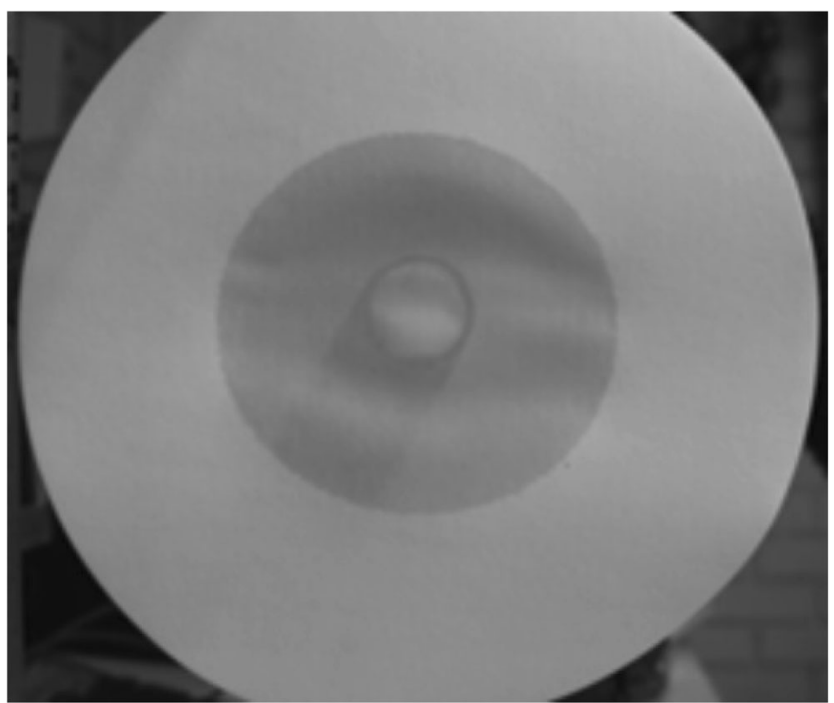

Fig. 3 Image of the spreading diameter on the filter paper for Triton X-100

\section{Results and Discussion: Radius of the Wetted Area Inside Porous Substrate}

Capillary flow inside the foam and liquid imbibition inside the porous substrate are described by dimensionless Eqs.
(5) and (31), respectively, have been solved analytically using COMSOL software finite element method on one dimensional regular grid. A backward differentiation formula was used to solve time-dependent variables and time stepping was free taken by the solver with initial step size of $10^{-20}$. Relative tolerance was set to $10^{-8}$, whereas absolute tolerance was set to $10^{-10}$. The values of foam/porous substrate systems parameters, $\mathrm{K}_{\mathrm{p}}, \Delta$, average $\mathrm{R}_{\mathrm{b}}, \varepsilon, \mu, \rho$, average pore size and initial liquid volume fraction, $\varphi_{\mathrm{i}}$, have been incorporated in simulations based on the experimental data as shown in Table 2 .

The imbibition into the porous substrate is characterised through measurements of wetted radius, $R(t)$, with initial value equal to the radius of the foam column $R(0)=R_{c}$ (see Fig. 1). Experimentally, spreading radius was accurately measured due to the symmetry of the circular shape of the wetted area (see Fig. 3).

Experimentally obtained time evolutions of spreading radius, $R(t)[\mathrm{cm}]$ inside porous layer are in a reasonable agreement with the theoretical predictions for all investigated surfactant solutions-filter paper systems, for both initial volume fractions of Triton-X-100 i.e. 20CMC and 50CMC and for filter papers of four different grades, i.e. grade 1,3,5 and 601, as shown in Figs. 4 and 5.
Fig. 4 Comparison between experimental and simulations results of time evolutions of spreading radius $\mathrm{R}(\mathrm{t})$ for a Triton X-100-20CMC-FPG1 b Triton X-100-20CMC-FPG3 c Triton X-100-20CMC-FPG5 and d Triton X-100-20CMC-FPG601
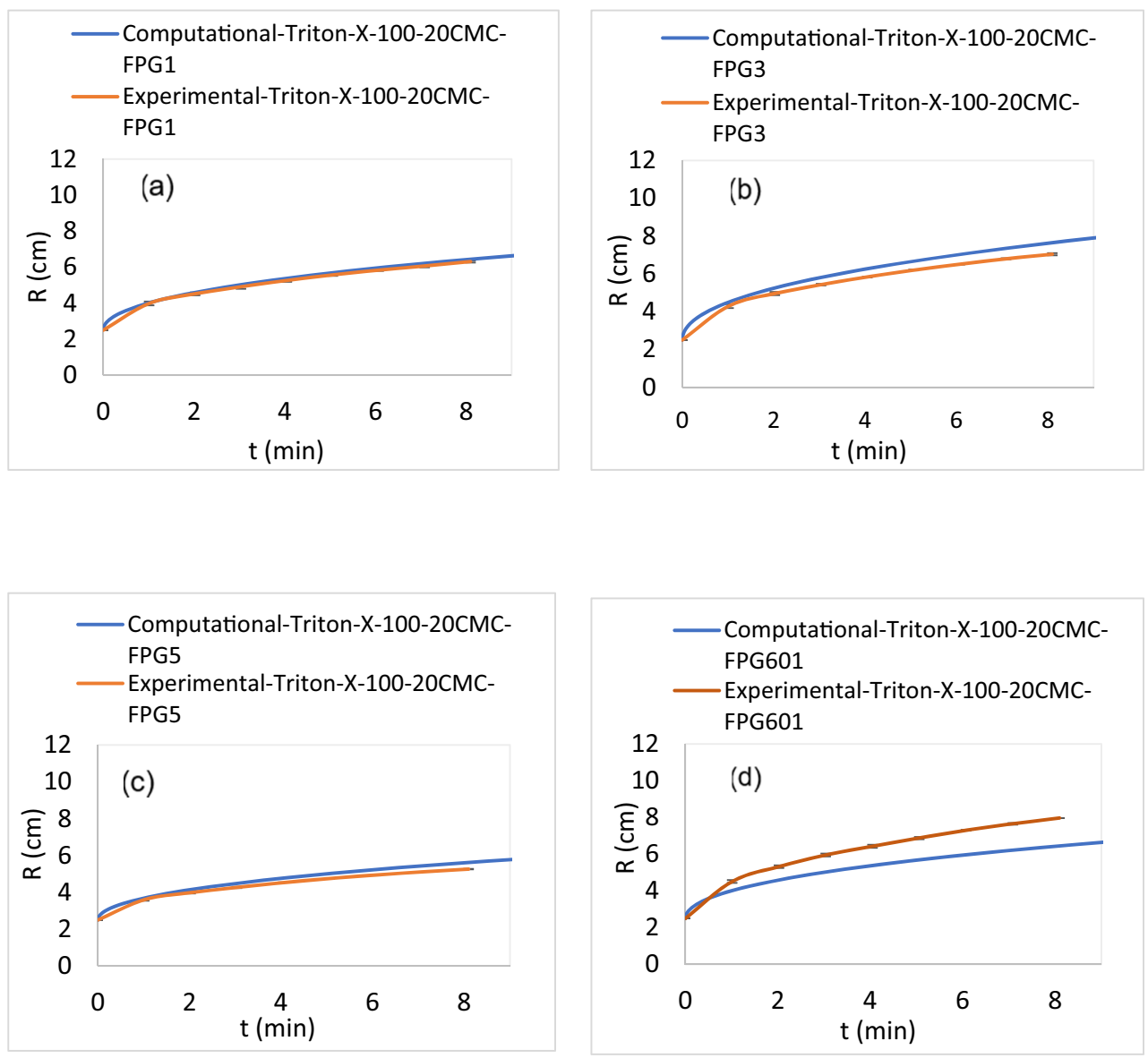
Fig. 5 Comparison between experimental and simulations results of time evolutions of spreading radius $\mathrm{R}(\mathrm{t})$ for a Triton X-100-50CMC-FPG1 b Triton X-100-50CMC-FPG3 c Triton X-100-50CMC-FPG5 and d Triton X-100-50CMC-FPG601
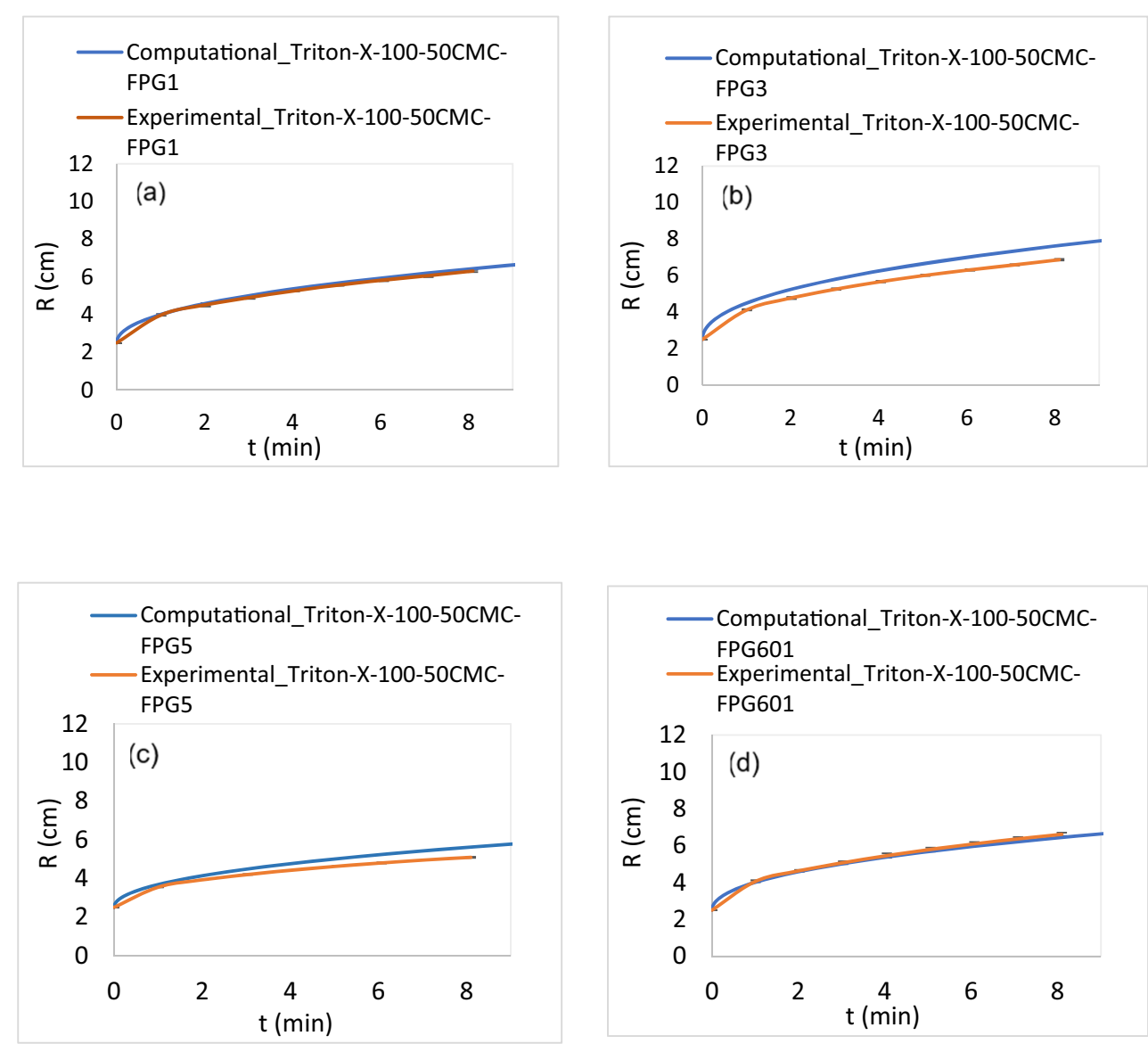

If $\Delta P>0$ then liquid from the Plateau border will penetrate the porous substrate. Spreading radius, $R(t)$, gradually increases until final liquid volume fraction at the foam/porous layer interface, $\varphi_{f}$, is reached (see Eq. (19)), when the capillary drainage/imbibition process is completed, and there is no further liquid penetration into the porous substrate. When $\varphi_{f}$ is reached, as expressed by Eq. (16), the pressure inside the Plateau channels and the pressure inside the porous layer equilibrate.

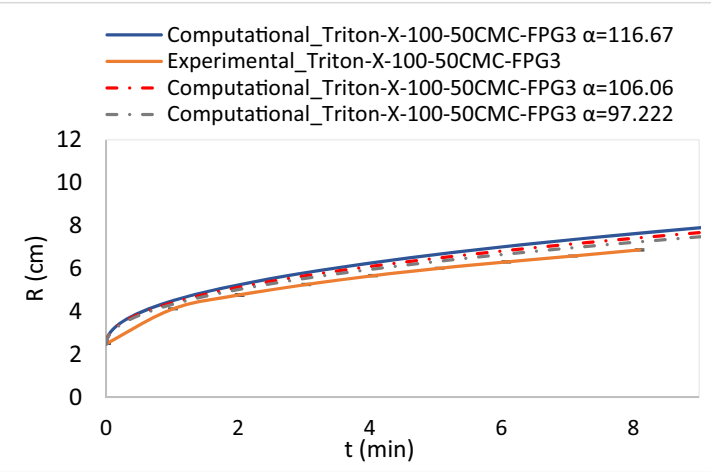

Fig. 6 Comparison between experimental and simulations results of time evolutions of spreading radius $\mathrm{R}(\mathrm{t})$ for Triton $\mathrm{X}-100-50 \mathrm{CMC}-\mathrm{FPG} 3$ for different values of dimensionless parameter $\alpha$, as expressed by Eq. (17)
Calculations show that dimensionless parameter $\alpha$, as expressed by Eq. (17), has shown a considerable effect to the rate of spreading $R(t)$. Any increase of parameter $\alpha$ results to an increase of the rate of spreading, $R(t)$. Parameter $\alpha$ is a function of bubble radious, $R_{b}$, and the pore radius of the porous layer, $R_{p m}$, and its value depends on the error in experimentall measurements of both parameters. Dimensionless parameters $\varphi_{i}, \varepsilon$ and $\zeta_{\Delta}$ showed a smaller effect to the radius of spreading, $R(t)$. The effect of small changes of dimensionless parameter $\alpha$ for Triton X-100-50CMCFPG3 is presented in Fig. 6.

Figure 6 shows that experimental error in calculation of both bubble radius, $R_{b}$, and the pore radius of the porous layer, $R_{p m}$, which influence the calculated dependency $R(t)$ through the parameter $\alpha$. Even a small variation of the latter parameter can improve agreement between the calculated and experimental dependences.

\section{Conclusions}

A new method of foams drying under microgravity conditions is suggested. A theory of the method was developed and compared with experimental measurements of the process. 
Comparison of the experimental data with a theoretical prediction are in very reasonable agreement.

Acknowledgements This research was supported by CoWet EU grant, grant from Proctor \& Gamble, Brussels; MAP EVAPORATION and PASTA grants from European Space Agency.

Open Access This article is distributed under the terms of the Creative Commons Attribution 4.0 International License (http:// creativecommons.org/licenses/by/4.0/), which permits unrestricted use, distribution, and reproduction in any medium, provided you give appropriate credit to the original author(s) and the source, provide a link to the Creative Commons license, and indicate if changes were made.

\section{References}

Arjmandi-Tash, O., Kovalchuk, N., Trybala, A., Starov, V.: Foam drainage placed on a porous substrate. Soft Matter. 11, 3643-3652 (2015). https://doi.org/10.1039/c5sm00377f

Arjmandi-Tash, O., Trybala, A., Mahdi, F.M., Kovalchuk, N.M., Starov, V.: Foams built up by non-Newtonian polymeric solutions: Free drainage. Colloids Surfaces A Physicochem. Eng. Asp. 521, 112120 (2017). https://doi.org/10.1016/j.colsurfa.2016.07.097

Banhart, J.: Foam Metal: The Recipe. Europhys. News. 30, (1999). https://doi.org/10.1007/s007700050113

Banhart, J., Weaire, T.D.: On the road again: metal foams find favor. Am. Inst. Phys. 37-42 (2002)

Bureiko, A., Arjmandi-Tash, O., Kovalchuk, N., Trybala, A., Starov, V.: Interaction of foam with a porous medium: Theory and calculations. Eur. Phys. J. Spec. Top. 224, 459-471 (2015). https://doi.org/10. 1140/epjst/e2015-02374-2

Camlab (2019) Quantitative Ashless Filter Papers. Retrieved from https:// www.camlab.co.uk/quantitative-ashless-filter-papers-c3583.aspx\#

Caps, H., Soyez, G., Chevalier, M.-L., Decauwer, H., Ausloos, M., Vandewalle, N.: Foam imbibition in microgravity. Eur. Phys. J. B Condens. Matter. 33, 115-119 (2003). https://doi.org/10.1140/epjb/ e2003-00148-9

Carrier, V., Destouesse, S., Colin, A.: Foam drainage: A film contribution? Phys. Rev. E - Stat. Physics, Plasmas, Fluids, Relat. Interdiscip. Top. 65, 1-9 (2002). https://doi.org/10.1103/ PhysRevE.65.061404

Cox, S.J., Weaire, D. \& Verbist, G. Eur. Phys. J. B.: Comment on "Foam imbibition in microgravity. An experimental study" by H. Caps, H. Decauwer, M.-L. Chevalier, G. Soyez, M. Ausloos and N. Vandewalle. The European Physical Journal B - Condensed Matter and Complex Systems. 40, 119 (2004). https://doi.org/10.1140/epjb/ e2004-00246-2

Cox, S.J., Weaire, D., Hutzler, S., Murphy, J., Phelan, R., Verbist, G.: Applications and generalizations of the foam drainage equation. Proc. R. Soc. London. Ser. A Math. Phys. Eng. Sci. 456, $2441-$ 2464 (2000). https://doi.org/10.1098/rspa.2000.0620

Divinis, N., Karapantsios, T.D., de Bruijn, R., Kostoglou, M., Bontozoglou, V., Legros, J.C.: Lateral motion and interaction of gas bubbles growing over spherical and plate heaters. Microgravity - Sci. Technol. 18, 204-209 (2006). https://doi.org/ $10.1007 / \mathrm{bf0} 2870410$

Dubois, F., Joannes, L., Dupont, O., Dewandel, J.L., Legros, J.C.: An integrated optical set-up for fluid-physics experiments under microgravity conditions. Meas. Sci. Technol. 10, 934-945 (1999). https:// doi.org/10.1088/0957-0233/10/10/314

Exerowa, D., Kruglyakov, P.: Foam and foam films: theory, experiment, application. Elsevier Science B.V, Amsterdam (1998)
Farajzadeh, R., Andrianov, A., Krastev, R., Hirasaki, G.J., Rossen, W.R.: Foam-oil interaction in porous media: Implications for foam assisted enhanced oil recovery. Adv. Colloid Interf. Sci. 183-184, 1-13 (2012). https://doi.org/10.1016/j.cis.2012.07.002

Goldfarb, I., Kahn, K.B., Schreiber, I.: Izv. Akad. Nauk SSSR. 2, (1987)

Goldshtein, V., Goldfarb, I., Schreiber, I.: Multiphase Flow at press. Int. J. (1996)

Grassia, P., Cilliers, J.J., Neethling, S.J., Ventura-Medina, E.: Quasi-onedimensional foam drainage. Eur. Phys. J. E. 6, 325-348 (2001). https://doi.org/10.1007/s10189-001-8047-3

Hart, D., Hansen, N., Legros, J.-C., Schramm, L.L.: Microgravity, industry related research for oil recovery. Am. Inst. Phys. 387, (1997). https://doi.org/10.1063/1.52077

Koehler, S.A., Hilgenfeldt, S., Stone, H.A.: Generalized view of foam drainage: Experiment and theory. Langmuir. 16, 6327-6341 (2000). https://doi.org/10.1021/la9913147

Kruglyakov, P.M., Karakashev, S.I., Nguyen, A.V., Vilkova, N.G.: Foam drainage. Curr. Opin. Colloid Interface Sci. 13, 163-170 (2008). https://doi.org/10.1016/j.cocis.2007.11.003

Prodi, F., Santachiara, G., Travaini, S., Vedernikov, A., Dubois, F., Minetti, C., Legros, J.C.: Measurements of phoretic velocities of aerosol particles in microgravity conditions. Atmos. Res. 82, 183189 (2006). https://doi.org/10.1016/j.atmosres.2005.09.010

Saint-Jalmes, A., Cox, S.J., Marze, S., Safouane, M., Langevin, D., Weaire, D.: Experiments and simulations of liquid imbibition in aqueous foams under microgravity. Microgravity - Sci. Technol. 18, 108-111 (2006). https://doi.org/10.1007/BF02870391

Schramm LL (1994) Foams: Fundamentals and applications in the petroleum industry. ACS Advances in Chemistry Series No. 242 (Am. Chem. Soc.), Washington DC

Schramm, L.L.: Emulsions, Foams, and Suspensions: Fundamentals and Applications. Wiley-VCH, Weinheim (2005)

Sigma-Aldrich (MERCK) (n.d.) Product results, https://www. sigmaaldrich.com/catalog/search?term=FILTER+ PAPERS\&interface $=\mathrm{All} \& \mathrm{~N}=0 \&$ mode $=$ matchpartialmax $\&$ lang $=$ en\&region $=\mathrm{GB} \&$ focus $=$ product

Sigma-Aldrich (MERCK) (2019) Triton ${ }^{\mathrm{TM}} \mathrm{X}-100$, https://www. sigmaaldrich.com/catalog/product/sial/x100?lang=en\&region=GB

Smirnov, N.N., Legros, J.C., Nikitin, V.F., Istasse, E., Schramm, L., Wassmuth, F., Hart, D.: Filtration in artificial porous media and natural sands under microgravity conditions. Microgravity - Sci. Technol. 14, 3-28 (2003). https://doi.org/10.1007/BF02870312

Smirnov, N.N., Nikitin, V.F., Ivashnyov, O.E., Maximenko, A., Thiercelin, M., Vedernikov, A., Scheid, B., Legros, J.C.: Microgravity investigations of instability and mixing flux in frontal displacement of fluids. Microgravity Sci. Technol. 15, 35-51 (2005). https://doi.org/10.1007/BF02870957

Smirnov, N.N., Nikitin, V.F., Legros, J.C., Istasse, E., Schramm, L., Wassmuth, F.: Microgravity investigations of capillary-driven imbibition and drainage in inhomogeneous porous media. Acta Astronaut. 54, 39-52 (2004). https://doi.org/10.1016/S00945765(02)00278-3

Starov, V.M., Kostvintsev, S.R., Sobolev, V.D., Velarde, M.G., Zhdanov, S.A.: Spreading of Liquid Drops over Dry Porous Layers: Complete Wetting Case. Langmuir. 252, 397-408 (2002)

Sun, Q., Tan, L., Wang, G.: Liquid foam drainage: an overview. Int. J. Mod. Phys. B. 22, 2333-2354 (2008)

Touzet, M., Galliero, G., Lazzeri, V., Saghir, M.Z., Montel, F., Legros, J.C.: Thermodiffusion: From microgravity experiments to the initial state of petroleum reservoirs. Comptes Rendus - Mec. 339, 318-323 (2011). https://doi.org/10.1016/j.crme.2011.03.008

Van Vaerenbergh, S., Legros, J.C.: Soret Coefficients of Organic Solutions Measured in the Microgravity SCM Experiment and by the Flow and Bénard Cells. J. Phys. Chem. B. 102, 4426-4431 (1998). https://doi.org/10.1021/jp9802329 
Vandewalle, N., Caps, H., Delon, G., Saint-Jalmes, A., Rio, E., Saulnier, L., Adler, M., Biance, A.L., Pitois, O., Addad, S.C., Hohler, R., Weaire, D., Hutzler, S., Langevin, D.: Foam stability in microgravity. J. Phys. Conf. Ser. 327, (2011). https://doi.org/10.1088/17426596/327/1/012024

Verbist, G., Weaire, D., Kraynik, A.M.: The foam drainage equation. J. Phys. Condens. Matter. 8, 3715-3731 (1996). https://doi.org/10. 1088/0953-8984/8/21/002

Wang, Z., Narsimhan, G.: Model for Plateau border drainage of powerlaw fluid with mobile interface and its application to foam drainage. J. Colloid Interface Sci. 300(1), 327-337 (2006). https://doi.org/10. 1016/j.jcis.2006.03.023
Weaire, D.L., Hutzler, S.: The Physics of Foams. Oxford University Press, Oxford (1999)

Wierenga, P.A., Gruppen, H.: New views on foams from protein solutions. Curr. Opin. Colloid Interface Sci. 15, 365-373 (2010). https:// doi.org/10.1016/j.cocis.2010.05.017

Zhao, Y., Jones, S.A., Brown, M.B.: Dynamic foams in topical drug delivery. Jpp. 62, 678-684 (2010). https://doi.org/10.1211/jpp/62. 06.0003

Publisher's Note Springer Nature remains neutral with regard to jurisdictional claims in published maps and institutional affiliations. 